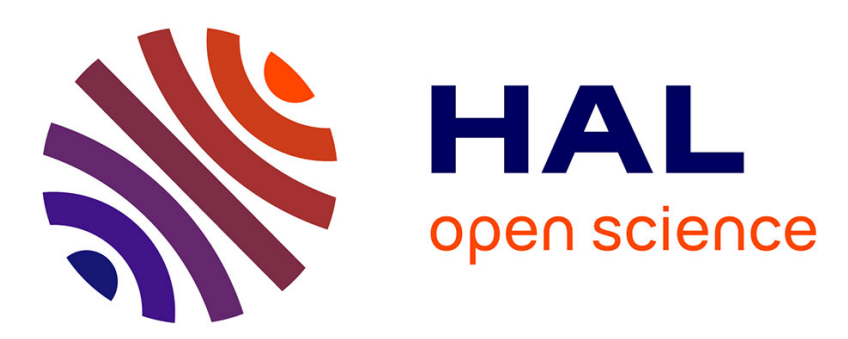

\title{
Micromechanical modelling of twinning in polycrystalline materials: Application to magnesium
}

Charles Mareau, Mark R Daymond

\section{To cite this version:}

Charles Mareau, Mark R Daymond. Micromechanical modelling of twinning in polycrystalline materials: Application to magnesium. International Journal of Plasticity, 2016, 85, pp.156-171. 10.1016/j.ijplas.2016.07.007 . hal-02486161

\section{HAL Id: hal-02486161 \\ https://hal.science/hal-02486161}

Submitted on 20 Feb 2020

HAL is a multi-disciplinary open access archive for the deposit and dissemination of scientific research documents, whether they are published or not. The documents may come from teaching and research institutions in France or abroad, or from public or private research centers.
L'archive ouverte pluridisciplinaire HAL, est destinée au dépôt et à la diffusion de documents scientifiques de niveau recherche, publiés ou non, émanant des établissements d'enseignement et de recherche français ou étrangers, des laboratoires publics ou privés. 


\title{
Micromechanical modelling of twinning in polycrystalline materials: Application to magnesium
}

\author{
Charles Mareau ${ }^{\mathrm{a}}$, Mark R. Daymond ${ }^{\mathrm{b}}$ \\ a Arts et Métiers ParisTech, Campus d'Angers, LAMPA, 2 boulevard du Ronceray, \\ 49035 Angers Cedex 1, France \\ ${ }^{\mathrm{b}}$ Dept. of Mechanical and Materials Engineering, Nicol Hall, 60 Union Street, \\ Kingston, KrL 3N6, Ontario, Canada
}

\begin{abstract}
In this work, a crystal plasticity constitutive model is proposed to describe the mechanical behavior of metallic materials for which twinning plays a significant role in the deformation process. Constitutive relations are obtained from a micromechanical approach that explicitly considers the interactions between twinned and untwinned domains. Then, based on a thermodynamical analysis of the problem, a new expression for the driving force for the expansion of twinned domains is proposed. Finally, to account for the polycrystalline nature of metallic materials, the constitutive model is implemented in a FFT spectral solver.

In the second part of this paper, the model is used to study the mechanical behavior of a AZ31 magnesium alloy under compression, for which a significant amount of experimental data is available in the literature. The comparison between numerical and experimental data allows for discussion of the influence of the different deformation modes on the development of both crystallographic texture and lattice strains. The evolution of lattice strains is found to be largely influenced by the internal stress redistribution process associated with the expansion of twinned domains. Also, the polycrystalline plasticity model provides a correct description of how the morphological texture is strongly altered during the deformation process due to the important activity of twinning systems.
\end{abstract}

Key words: A. Microstructures, A. Twinning, B. Polycrystalline material, B. Crystal plasticity, C. FFT method

Email addresses: charles.mareau@ensam.eu (Charles Mareau), daymond@queensu.ca (Mark R. Daymond). 


\section{Introduction}

For some metallic alloys (e.g. magnesium alloys, zirconium alloys, austenitic steels), mechanical behavior is strongly impacted by the coexistence of mechanical twinning and crystallographic slip deformation modes. While the crystal plasticity framework (Asaro and Needleman, 1985; Cuitiño and Ortiz, 1992; Rashid and Nemat-Nasser, 1990) provides a convenient way of introducing the influence of crystallographic slip in constitutive models, the incorporation of deformation twinning within such models is not trivial. Indeed, in order to include the role of deformation twinning in a consistent manner, some specific features of twinning deformation modes have to be considered:

- First, because of the abrupt crystallographic reorientation process associated with twinning, significant texture evolution is often observed during a deformation process (Brown et al., 2005; Vercammen et al., 2004; Xu et al., 2009). As a result, for polycrystalline aggregates, the anisotropic nature of macroscopic properties is strongly dependent on the crystallographic orientations of twinned domains.

- Second, as mentioned by Remy (1981) and Basinski et al. (1997), during a deformation process, deformation twinning results in the formation of additional boundaries that provide a significant contribution to strain hardening. The mechanical behavior of materials for which twinning plays an important role is thus governed by a strong coupling between slip and twinning deformation modes.

- Third, depending on loading conditions, twinning may contribute importantly to the plastic deformation process because the expansion of twinned domains involves the accumulation of large plastic shear strains at the grain scale. The macroscopic behavior is therefore very sensitive to the growth rate of twinned domains.

- Finally, different diffraction studies have demonstrated that twinning is accompanied by a significant redistribution of internal stresses (Clausen et al., 2008; Wu et al., 2008; Xu et al., 2009). Indeed, because the local behavior of individual crystals is influenced by their orientation, the reorientation process associated with twinning results in some complex load sharing mechanisms that affect the macroscopic behavior.

To account for these aspects of deformation twinning in constitutive relations, different strategies have been adopted. Using the self-consistent approximation, many efforts have been made to incorporate twinning in polycrystalline plasticity models. For instance, to investigate the evolution of texture in zirconium and magnesium alloys, some extensions of the viscoplastic self-consistent model of Lebensohn and Tomé (1993) have been proposed (Capolungo et al., 2009a; Knezevic et al., 2015; Proust et al., 2007). However, by construction, such models do not readily allow the evaluation of internal stresses be- 
cause elastic strains are not considered. To circumvent this limitation, different elasto-(visco)plastic formulations have been developed. From a micromechanical approach considering twins as ellipsoidal inclusions, Cherkaoui (2003) proposed a set of constitutive relations to investigate the behavior of f.c.c. metals. Shiekhelsouk et al. (2009) developed a physically based model where the resistance opposed by twin interfaces to dislocation glide is explicitly considered. Clausen et al. (2008) constructed a rate-independent self-consistent model to study the development of lattice strains and texture in a magnesium alloy. Nevertheless, mostly because the interactions between twinned and untwinned domains are ignored, the approach of Clausen et al. (2008), which has been reformulated in a rate-dependent framework by Mareau and Daymond (2011), does not completely succeeds in matching the experimental observations. To better describe these interactions, Juan et al. (2014) proposed a self-consistent formulation based on a double inclusion formalism. Although this strategy allows the investigation of the competition between slip and twinning deformation modes, the moving boundary aspect of the problem of twin growth is not considered in the approach of Juan et al. (2014).

Though some efforts have been made to include the effect stress field fluctuations on twin variant selection (Niezgoda et al., 2014; Zecevic et al., 2015), selfconsistent formulations do not explicitly account for the intragranular stress and strain field gradients. To circumvent this limitation, several attempts have been made at incorporating deformation twinning within crystal plasticity based finite element models (Abdolvand et al., 2012; Cheng and Ghosh, 2015; Kalidindi, 2001; Staroselsky and Anand, 2003). In a large strain formalism, Kalidindi (2001) has developed a constitutive model that uses a simple mixture rule to include the contribution of both twinning and slip deformation modes. While this type of approach has been successful in describing texture evolution, some aspects of the development of internal stresses are not well described, mostly because the elastic strain field is assumed to be the same in the twinned and untwinned domains. Cheng and Ghosh (2015) proposed a non-local crystal plasticity model to investigate the twin nucleation process in magnesium alloys. Also, as discussed by Knezevic et al. (2016), different strategies have been employed to explicitly model discrete twinned domains (Ardeljan et al., 2015; Kumar et al., 2015; Zhang et al., 2008). Indeed, Zhang et al. (2008) and Kumar et al. (2015) have used either the finite element method or FFT-based techniques to investigate the internal stress field produced by an isolated deformation twin. A more complex numerical procedure has recently been proposed by Ardeljan et al. (2015) to deal with twin propagation and twin growth in crystal plasticity based finite element models. The major drawback of these approaches is that the twin morphology needs to be known a priori.

In this work, a method for considering the influence of deformation twinning in polycrystalline plasticity models is presented. The first part of this paper is 
dedicated to the description of the proposed model. Adopting a similar strategy as Cherkaoui (2003), the local constitutive relations are derived from a micromechanical analysis of the problem. Within a thermodynamically consistent framework, the proposed formulation provides an explicit description of the interactions between twinned and untwinned domains. To account for intragranular heterogeneities, the constitutive relations are then implemented in a spectral (FFT) solver that exploits the method originally proposed by Moulinec and Suquet (1998). In the second part, to demonstrate the relevance of the proposed constitutive relations, the model is used to investigate the behavior of an AZ31 magnesium alloy, for which an extensive experimental dataset has been obtained by Clausen et al. (2008). During the mechanical testing of a magnesium alloy sample, Clausen et al. (2008) carried out neutron diffraction measurements to study the development of lattice strains and crystallographic texture. The direct comparison of numerical results with experimental measurements thus allows for better understanding the contribution of the different deformation modes, the evolution of internal stresses, the formation of intragranular lamellar microstructures and the reorientation processes.

\section{Model description}

\subsection{Constitutive model}

In this section, a constitutive model, which is developed for an element of volume $v$ with external boundary $\partial v$, is proposed. The volume element $v$ is an intragranular domain for which plastic deformation possibly results from both slip and twinning deformation modes. For a such volume element, the constitutive model aims at connecting the stress tensor $\overline{\boldsymbol{\sigma}}$ to the strain tensor $\bar{\varepsilon}$ with an appropriate set of internal variables.

As shown in figure 1, to account for the contribution of twinning, the volume element is decomposed into two domains separated by a planar interface of area $a(t)$ : an untwinned domain (superscript $u$ ) of volume $v^{u}(t)$ and a twinned domain (superscript $t$ ) of volume $v^{t}(t)$. Based on this decomposition, the stress, strain and rotation fields are assumed to be piecewise uniform within the volume element. Thus, with an indicator function $\eta(\boldsymbol{x}, t)$ taking a unity value within a twinned domain and a zero value elsewhere, the stress, strain and rotation tensors at position $\boldsymbol{x}$ are written:

$$
\begin{aligned}
\boldsymbol{\sigma}(\boldsymbol{x}, t) & =\eta(\boldsymbol{x}, t) \boldsymbol{\sigma}^{t}(t)+(1-\eta(\boldsymbol{x}, t)) \boldsymbol{\sigma}^{u}(t) \\
\boldsymbol{\varepsilon}(\boldsymbol{x}, t) & =\eta(\boldsymbol{x}, t) \boldsymbol{\varepsilon}^{t}(t)+(1-\eta(\boldsymbol{x}, t)) \boldsymbol{\varepsilon}^{u}(t) \\
\boldsymbol{\omega}(\boldsymbol{x}, t) & =\eta(\boldsymbol{x}, t) \boldsymbol{\omega}^{t}(t)+(1-\eta(\boldsymbol{x}, t)) \boldsymbol{\omega}^{u}(t)
\end{aligned}
$$




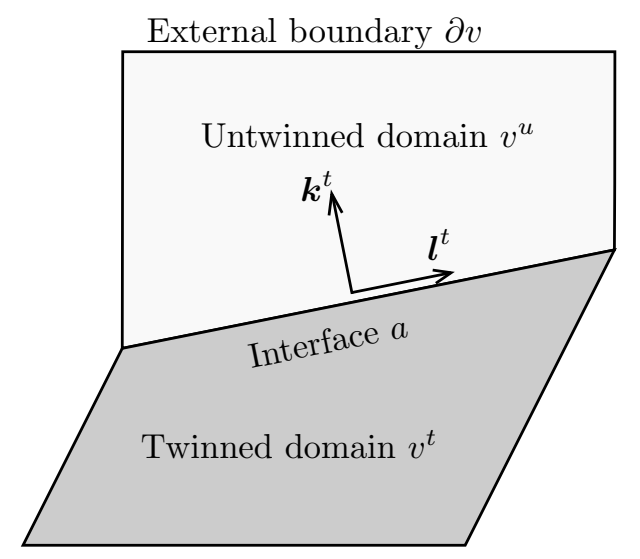

Figure 1. Schematic representation of a twinned volume element.

The values of the stress, strain and rotation fields within the untwinned domain (and for the twinned domain) are denoted by $\boldsymbol{\sigma}^{u}(t), \boldsymbol{\varepsilon}^{u}(t)$ and $\boldsymbol{\omega}^{u}(t)$ (respectively $\boldsymbol{\sigma}^{t}(t), \boldsymbol{\varepsilon}^{t}(t)$ and $\boldsymbol{\omega}^{t}(t)$ ). In the following, the dependence with time and position will be omitted unless needed.

\subsubsection{Crystal plasticity framework}

Under the infinitesimal deformation framework, the strain and rotation tensors associated with the untwinned and twinned domains are divided into elastic (superscript $e$ ) and plastic (superscript $p$ ) contributions:

$$
\begin{aligned}
\varepsilon^{u} & =\varepsilon^{e, u}+\varepsilon^{p, u} \\
\boldsymbol{\omega}^{u} & =\boldsymbol{\omega}^{e, u}+\boldsymbol{\omega}^{p, u} \\
\varepsilon^{t} & =\boldsymbol{\varepsilon}^{e, t}+\boldsymbol{\varepsilon}^{p, t} \\
\boldsymbol{\omega}^{t} & =\boldsymbol{\omega}^{e, t}+\boldsymbol{\omega}^{p, t}
\end{aligned}
$$

Assuming a linear relation between the elastic strain tensors $\left(\varepsilon^{e, u}\right.$ and $\left.\varepsilon^{e, t}\right)$ and the corresponding stress tensors $\left(\boldsymbol{\sigma}^{u}\right.$ and $\left.\boldsymbol{\sigma}^{t}\right)$, one obtains:

$$
\begin{gathered}
\boldsymbol{\sigma}^{u}=\boldsymbol{c}^{u}:\left(\varepsilon^{u}-\varepsilon^{p, u}\right) \\
\boldsymbol{\sigma}^{t}=\boldsymbol{c}^{t}:\left(\varepsilon^{t}-\varepsilon^{p, t}\right)
\end{gathered}
$$

where $\boldsymbol{c}^{u}$ (or $\boldsymbol{c}^{t}$ ) is the elastic stiffness tensor of the untwinned domain (respectively for the twinned domain).

Within a crystal plasticity framework, a set of $s$ slip systems is associated to each of the untwinned and twinned domains. For the untwinned domain (or twinned domain), a given slip system (say $\alpha$, with $\alpha=1$ to $s$ ) is defined as the combination of a slip plane of normal $\boldsymbol{n}^{\alpha, u}$ (respectively $\boldsymbol{n}^{\alpha, t}$ ) and a slip direction $\boldsymbol{p}^{\alpha, u}$ (respectively $\boldsymbol{p}^{\alpha, t}$ ). The plastic shear strain associated with the $\alpha$ th slip system of the untwinned domain (or twinned domain) is 
denoted by $\gamma^{\alpha, u}$ (respectively $\gamma^{\alpha, t}$ ). For the untwinned and twinned domains, the corresponding plastic strain and rotation tensors are connected to the plastic shear strains according to:

$$
\begin{gathered}
\varepsilon^{p, u}=\frac{1}{2} \sum_{\alpha}\left(\boldsymbol{p}^{\alpha, u} \otimes \boldsymbol{n}^{\alpha, u}+\boldsymbol{n}^{\alpha, u} \otimes \boldsymbol{p}^{\alpha, u}\right) \gamma^{\alpha, u} \\
\boldsymbol{\omega}^{p, u}=\frac{1}{2} \sum_{\alpha}\left(\boldsymbol{p}^{\alpha, u} \otimes \boldsymbol{n}^{\alpha, u}-\boldsymbol{n}^{\alpha, u} \otimes \boldsymbol{p}^{\alpha, u}\right) \gamma^{\alpha, u} \\
\boldsymbol{\varepsilon}^{p, t}=\frac{1}{2} \sum_{\alpha}\left(\boldsymbol{p}^{\alpha, t} \otimes \boldsymbol{n}^{\alpha, t}+\boldsymbol{n}^{\alpha, t} \otimes \boldsymbol{p}^{\alpha, t}\right) \gamma^{\alpha, t}+\boldsymbol{\varepsilon}^{t w, t} \\
\boldsymbol{\omega}^{p, t}=\frac{1}{2} \sum_{\alpha}\left(\boldsymbol{p}^{\alpha, t} \otimes \boldsymbol{n}^{\alpha, t}-\boldsymbol{n}^{\alpha, t} \otimes \boldsymbol{p}^{\alpha, t}\right) \gamma^{\alpha, t}+\boldsymbol{\omega}^{t w, t}
\end{gathered}
$$

where $\boldsymbol{\varepsilon}^{t w, t}$ and $\boldsymbol{\omega}^{t w, t}$ represent the additional contributions due to twinning. These contributions are expressed as a function of the characteristic twin shear strain $\gamma^{t w, t}$ as follows:

$$
\begin{aligned}
\varepsilon^{t w, t} & =\frac{1}{2}\left(\boldsymbol{l}^{t} \otimes \boldsymbol{k}^{t}+\boldsymbol{k}^{t} \otimes \boldsymbol{l}^{t}\right) \gamma^{t w, t} \\
\boldsymbol{\omega}^{t w, t} & =\frac{1}{2}\left(\boldsymbol{l}^{t} \otimes \boldsymbol{k}^{t}-\boldsymbol{k}^{t} \otimes \boldsymbol{l}^{t}\right) \gamma^{t w, t}
\end{aligned}
$$

where $\boldsymbol{l}^{t}$ is the twin direction and $\boldsymbol{k}^{t}$ is the twin plane normal (see figure 1).

\subsubsection{Partition method}

For the volume element $v$, the size of the twinned domain is represented by the twin volume fraction $\phi^{t}(t)$ which is given by:

$$
\phi^{t}=\frac{1}{v} \int_{v} \eta d v=\frac{v^{t}}{v}
$$

Since two domains coexist within the volume element, the corresponding stress, strain and rotation tensors $(\overline{\boldsymbol{\sigma}}, \overline{\boldsymbol{\varepsilon}}$ and $\overline{\boldsymbol{\omega}})$ have to be partitioned between the untwinned and twinned domains. According to the classical averaging conditions of homogenization theory, the partition must satisfy the following equalities at each time $t$ :

$$
\begin{aligned}
\overline{\boldsymbol{\sigma}} & =\frac{1}{v} \int_{v} \boldsymbol{\sigma} d v=\phi^{t} \boldsymbol{\sigma}^{t}+\left(1-\phi^{t}\right) \boldsymbol{\sigma}^{u} \\
\overline{\boldsymbol{\varepsilon}} & =\frac{1}{v} \int_{v} \boldsymbol{\varepsilon} d v=\phi^{t} \boldsymbol{\varepsilon}^{t}+\left(1-\phi^{t}\right) \boldsymbol{\varepsilon}^{u} \\
\overline{\boldsymbol{\omega}} & =\frac{1}{v} \int_{v} \boldsymbol{\omega} d v=\phi^{t} \boldsymbol{\omega}^{t}+\left(1-\phi^{t}\right) \boldsymbol{\omega}^{u}
\end{aligned}
$$


This set of relations is however not sufficient to uniquely determine the stress, strain and rotation tensors within the untwinned and twinned domains. Additional constraints are introduced by considering the interface between both domains to be perfect. For a perfect interface, the continuity of the traction vector imposes:

$$
\boldsymbol{\sigma}^{u} \cdot \boldsymbol{k}^{t}=\boldsymbol{\sigma}^{t} \cdot \boldsymbol{k}^{t}=\overline{\boldsymbol{\sigma}} \cdot \boldsymbol{k}^{t}
$$

Using relations (8) and (9), the above equation is reformulated:

$$
\left(\boldsymbol{c}^{u}:\left(\boldsymbol{\varepsilon}^{u}-\boldsymbol{\varepsilon}^{p, u}\right)\right) \cdot \boldsymbol{k}^{t}=\left(\boldsymbol{c}^{t}:\left(\boldsymbol{\varepsilon}^{t}-\boldsymbol{\varepsilon}^{p, t}\right)\right) \cdot \boldsymbol{k}^{t}=\left(\overline{\boldsymbol{c}}:\left(\overline{\boldsymbol{\varepsilon}}-\overline{\boldsymbol{\varepsilon}}^{p}\right)\right) \cdot \boldsymbol{k}^{t}
$$

where $\overline{\boldsymbol{c}}$ and $\overline{\boldsymbol{\varepsilon}}^{p}$ are respectively the effective elastic stiffness tensor and the effective plastic strain tensor of the volume element. Also, the compatibility of the displacement gradient field provides supplementary equations:

$$
\begin{aligned}
\boldsymbol{\varepsilon}^{u}:\left(\boldsymbol{l}^{t} \otimes \boldsymbol{l}^{t}\right) & =\varepsilon^{t}:\left(\boldsymbol{l}^{t} \otimes \boldsymbol{l}^{t}\right)=\overline{\boldsymbol{\varepsilon}}:\left(\boldsymbol{l}^{t} \otimes \boldsymbol{l}^{t}\right) \\
\varepsilon^{u}:\left(\boldsymbol{m}^{t} \otimes \boldsymbol{m}^{t}\right) & =\varepsilon^{t}:\left(\boldsymbol{m}^{t} \otimes \boldsymbol{m}^{t}\right)=\overline{\boldsymbol{\varepsilon}}:\left(\boldsymbol{m}^{t} \otimes \boldsymbol{m}^{t}\right) \\
\boldsymbol{\varepsilon}^{u}:\left(\boldsymbol{l}^{t} \otimes \boldsymbol{m}^{t}\right) & =\varepsilon^{t}:\left(\boldsymbol{l}^{t} \otimes \boldsymbol{m}^{t}\right)=\overline{\boldsymbol{\varepsilon}}:\left(\boldsymbol{l}^{t} \otimes \boldsymbol{m}^{t}\right) \\
\boldsymbol{\omega}^{u}:\left(\boldsymbol{l}^{t} \otimes \boldsymbol{m}^{t}\right) & =\boldsymbol{\omega}^{t}:\left(\boldsymbol{l}^{t} \otimes \boldsymbol{m}^{t}\right)=\overline{\boldsymbol{\omega}}:\left(\boldsymbol{l}^{t} \otimes \boldsymbol{m}^{t}\right) \\
\left(\varepsilon^{u}+\boldsymbol{\omega}^{u}\right):\left(\boldsymbol{k}^{t} \otimes \boldsymbol{l}^{t}\right) & =\left(\varepsilon^{t}+\boldsymbol{\omega}^{t}\right):\left(\boldsymbol{k}^{t} \otimes \boldsymbol{l}^{t}\right)=(\overline{\boldsymbol{\varepsilon}}+\overline{\boldsymbol{\omega}}):\left(\boldsymbol{k}^{t} \otimes \boldsymbol{l}^{t}\right) \\
\left(\boldsymbol{\varepsilon}^{u}+\boldsymbol{\omega}^{u}\right):\left(\boldsymbol{k}^{t} \otimes \boldsymbol{m}^{t}\right) & =\left(\varepsilon^{t}+\boldsymbol{\omega}^{t}\right):\left(\boldsymbol{k}^{t} \otimes \boldsymbol{m}^{t}\right)=(\overline{\boldsymbol{\varepsilon}}+\overline{\boldsymbol{\omega}}):\left(\boldsymbol{k}^{t} \otimes \boldsymbol{m}^{t}\right)
\end{aligned}
$$

with $\boldsymbol{m}^{t}=\boldsymbol{k}^{t} \times \boldsymbol{l}^{t}$. At time $t$, for prescribed strain and rotation tensors $\bar{\varepsilon}$ and $\overline{\boldsymbol{\omega}}$, localization equations (21) to (27) allow for unique determination of the strain and rotation tensors $\varepsilon^{u}, \boldsymbol{\omega}^{u}, \boldsymbol{\varepsilon}^{t}$ and $\boldsymbol{\omega}^{t}$ associated with the untwinned and twinned domains. The corresponding stress tensors are obtained with relations (8) and (9). The effective properties of the volume element $\overline{\boldsymbol{c}}$ and $\overline{\boldsymbol{\varepsilon}}^{p}$ are calculated to enforce the fulfilment of averaging conditions (17) and (18).

The present strategy, which is quite similar to the lamellar structure model developed for single polysynthetically twinned crystals by Lebensohn et al. (1998), has two advantages. First, it relies on a consistent partitioning method that ensures the respect of averaging conditions (17) and (18). Second, the computational cost is quite low as no sophisticated numerical procedure is needed.

\subsubsection{Thermodynamics}

For the volume element $v$, the total free energy results from a volume contribution associated with the untwinned domain (with density $\psi^{u}$ ), a volume contribution associated with the twinned domain (with density $\psi^{t}$ ) and an interfacial contribution (with surface density $\chi^{i n t}$ ). The total free energy density 
$\bar{\psi}$ is thus obtained from the addition of the above contributions:

$$
\bar{\psi}=\phi^{t} \psi^{t}+\left(1-\phi^{t}\right) \psi^{u}+\frac{a}{v} \chi^{i n t}
$$

where $a / v$ is the ratio between the twin interface area $a$ and the volume $v$. The bulk free energy densities $\psi^{u}$ and $\psi^{t}$ can be defined with an appropriate set of state variables which consists of the infinitesimal strain tensors $\left(\varepsilon^{u}\right.$ and $\left.\varepsilon^{t}\right)$, the plastic shear strains $\left(\gamma^{\alpha, u}\right.$ and $\left.\gamma^{\alpha, t}\right)$ and the isotropic hardening variables (denoted by $q^{\alpha, u}$ and $\left.q^{\alpha, t}\right)^{1}$. Assuming a quadratic form for the bulk free energy densities $\psi^{u}$ and $\psi^{t}$ leads to:

$$
\begin{aligned}
& \psi^{u}=\frac{1}{2}\left(\varepsilon^{u}-\varepsilon^{p, u}\right): \boldsymbol{c}^{u}:\left(\varepsilon^{u}-\varepsilon^{p, u}\right)+\frac{1}{2} Q \sum_{\alpha} q^{\alpha, u} \sum_{\beta} H^{\alpha \beta} q^{\beta, u} \\
& \psi^{t}=\frac{1}{2}\left(\varepsilon^{t}-\varepsilon^{p, t}\right): \boldsymbol{c}^{t}:\left(\varepsilon^{t}-\varepsilon^{p, t}\right)+\frac{1}{2} Q \sum_{\alpha} q^{\alpha, t} \sum_{\beta} H^{\alpha \beta} q^{\beta, t}
\end{aligned}
$$

where $Q$ is the isotropic hardening modulus and $H$ is a symmetric and positivedefinite square matrix of size $s$ describing the interactions between different slip systems. To express the interface contribution to the total free energy density, an additional hardening internal variable $\lambda^{t}$ is introduced to represent the resistance to the expansion of the twinned domain. The interface contribution $\chi^{\text {int }}$ reads:

$$
\frac{a}{v} \chi^{i n t}=C \lambda^{t}\left(\phi^{t} \sum_{\alpha} q^{\alpha, t}+\left(1-\phi^{t}\right) \sum_{\alpha} q^{\alpha, u}\right)+\frac{1}{2} W \lambda^{t^{2}}
$$

where $W$ is a hardening modulus and $C$ is a material parameter controlling the interactions between slip and twinning deformation modes. If a more a complex type of interaction is observed, it is possible to include a slip system dependency for the $C$ parameter. The free energy change rate is provided by the time derivative of relation (28):

$$
\dot{\bar{\psi}}=\phi^{t} \dot{\psi}^{t}+\left(1-\phi^{t}\right) \dot{\psi}^{u}+\dot{\phi}^{t}\left(\psi^{t}-\psi^{u}\right)+\frac{d}{d t}\left(\frac{a}{v} \chi^{i n t}\right)
$$

For the volume element $v$, in the absence of volume forces, the density of power developed by external forces is:

$$
\bar{P}^{e}=\frac{1}{v} \int_{\partial v} \boldsymbol{t} \cdot \dot{\boldsymbol{u}} d s
$$

where $\boldsymbol{t}$ is the traction vector acting on the external boundary $\partial v$ and $\dot{\boldsymbol{u}}$ is the velocity field. The above relation can be reformulated by following the

1 Though kinematic hardening is not considered here, the introduction of a set of kinematic hardening variables does not bring any additional difficulty. 
strategy proposed by Cherkaoui (2003). Indeed, using the divergence theorem, the above relation becomes:

$$
\bar{P}^{e}=\frac{1}{v} \int_{v} \operatorname{div}(\boldsymbol{\sigma} \cdot \dot{\boldsymbol{u}}) d v+\frac{1}{v} \int_{a}\left(\boldsymbol{\sigma}^{u} \cdot \dot{\boldsymbol{u}}^{u}-\boldsymbol{\sigma}^{t} \cdot \dot{\boldsymbol{u}}^{t}\right) \cdot \boldsymbol{k}^{t} d s
$$

When inertia effects are neglected, the application of static equilibrium conditions leads to:

$$
\begin{aligned}
\operatorname{div}(\boldsymbol{\sigma} \cdot \dot{\boldsymbol{u}}) & =\boldsymbol{\sigma}: \operatorname{grad}(\dot{\boldsymbol{u}}) \\
& =\boldsymbol{\sigma}: \dot{\varepsilon}
\end{aligned}
$$

Also, using the continuity condition given by relation (20), we have:

$$
\left(\boldsymbol{\sigma}^{u} \cdot \dot{\boldsymbol{u}}^{u}-\boldsymbol{\sigma}^{t} \cdot \dot{\boldsymbol{u}}^{t}\right) \cdot \boldsymbol{k}^{t}=\frac{1}{2}\left(\left(\boldsymbol{\sigma}^{u}+\boldsymbol{\sigma}^{t}\right) \cdot \boldsymbol{k}^{t}\right) \cdot\left(\dot{\boldsymbol{u}}^{u}-\dot{\boldsymbol{u}}^{t}\right)
$$

According to Hadamard relation, the velocity jump across the twin interface is given by:

$$
\dot{\boldsymbol{u}}^{u}-\dot{\boldsymbol{u}}^{t}=\boldsymbol{k}^{t} \cdot\left(\varepsilon^{t}-\varepsilon^{u}+\boldsymbol{\omega}^{t}-\boldsymbol{\omega}^{u}\right) \boldsymbol{v}^{t} \cdot \boldsymbol{k}^{t}
$$

where $\boldsymbol{v}^{t}$ is the interface velocity vector. An alternative expression of the density of power developed by external forces is derived by combining relations (34) to (38):

$$
\bar{P}^{e}=\phi^{t} \boldsymbol{\sigma}^{t}: \dot{\boldsymbol{\varepsilon}}^{t}+\left(1-\phi^{t}\right) \boldsymbol{\sigma}^{u}: \dot{\boldsymbol{\varepsilon}}^{u}+\frac{1}{2 v}\left(\boldsymbol{\sigma}^{u}+\boldsymbol{\sigma}^{t}\right):\left(\varepsilon^{t}-\boldsymbol{\varepsilon}^{u}\right) \int_{a} \boldsymbol{v}^{t} \cdot \boldsymbol{k}^{t} d s
$$

The twin volume fraction rate is connected to the interface velocity vector $\boldsymbol{v}^{t}$ according to:

$$
\dot{\phi}^{t}=\frac{1}{v} \int_{a} \boldsymbol{v}^{t} \cdot \boldsymbol{k}^{t} d s
$$

The final expression of the density of power developed by external forces is obtained by introducing the above expression in relation (39):

$$
\bar{P}^{e}=\phi^{t} \boldsymbol{\sigma}^{t}: \dot{\boldsymbol{\varepsilon}}^{t}+\left(1-\phi^{t}\right) \boldsymbol{\sigma}^{u}: \dot{\boldsymbol{\varepsilon}}^{u}+\frac{1}{2} \dot{\phi}^{t}\left(\boldsymbol{\sigma}^{u}+\boldsymbol{\sigma}^{t}\right):\left(\varepsilon^{t}-\boldsymbol{\varepsilon}^{u}\right)
$$

The thermodynamic driving forces associated with the different internal variables of the constitutive model can be identified from the expression of the mechanical dissipation source. When inertia effects are excluded, the mechanical dissipation source $\bar{D}$ is obtained from the difference between the power of external forces and the free energy change rate. Thus, combining relations (29), (30), (31), (32) and (41) leads to the following expression of the mechanical 
dissipation source $\bar{D}$ :

$$
\begin{aligned}
\bar{D} & =\bar{P}^{e}-\dot{\bar{\psi}} \\
& =f^{t} \dot{\phi}^{t}-g^{t} \dot{\lambda}^{t}+\phi^{t} \sum_{\alpha} \tau^{\alpha, t} \dot{\gamma}^{\alpha, t}-\phi^{t} \sum_{\alpha} r^{\alpha, t} \dot{q}^{\alpha, t} \\
& +\left(1-\phi^{t}\right) \sum_{\alpha} \tau^{\alpha, u} \dot{\gamma}^{\alpha, u}-\left(1-\phi^{t}\right) \sum_{\alpha} r^{\alpha, u} \dot{q}^{\alpha, u}
\end{aligned}
$$

In the above expression, the different thermodynamic driving forces $\left(\tau^{\alpha, u}, \tau^{\alpha, t}\right.$, $r^{\alpha, u}, r^{\alpha, t}, f^{t}$ and $\left.g^{t}\right)$ depend on the internal variables $\left(\gamma^{\alpha, u}, \gamma^{\alpha, t}, q^{\alpha, u}, q^{\alpha, t}, \phi^{t}\right.$ and $\lambda^{t}$ ). More specifically, the driving forces for plastic slip are the applied shear stresses $\tau^{\alpha, u}$ and $\tau^{\alpha, t}$ acting on the different slip systems. For the $\alpha$ th slip system, the applied shear stresses $\tau^{\alpha, u}$ and $\tau^{\alpha, t}$ are given by the following projections of the stress tensors $\boldsymbol{\sigma}^{u}$ and $\boldsymbol{\sigma}^{t}$ :

$$
\begin{aligned}
\tau^{\alpha, u} & =\boldsymbol{p}^{\alpha, u} \cdot \boldsymbol{\sigma}^{u} \cdot \boldsymbol{n}^{\alpha, u} \\
\tau^{\alpha, t} & =\boldsymbol{p}^{\alpha, t} \cdot \boldsymbol{\sigma}^{t} \cdot \boldsymbol{n}^{\alpha, t}
\end{aligned}
$$

The resistance opposed by obstacles to plastic slip is represented by the shear stresses $r^{\alpha, u}$ and $r^{\alpha, t}$. The shear stresses $r^{\alpha, u}$ and $r^{\alpha, t}$ are connected to the isotropic hardening variables $q^{\alpha, u}$ and $q^{\alpha, t}$ according to:

$$
\begin{aligned}
& r^{\alpha, u}=Q \sum_{\beta} H^{\alpha \beta} q^{\beta, u}+C \lambda^{t} \\
& r^{\alpha, t}=Q \sum_{\beta} H^{\alpha \beta} q^{\beta, t}+C \lambda^{t}
\end{aligned}
$$

According to relations (43) and (44), isotropic hardening results not only from the interactions between slip systems but also from the interactions of a slip system with the twin interface.

The driving force for the growth of the twinned domain $f^{t}$ has the following expression $^{2}$ :

$$
f^{t}=\frac{1}{2}\left(\boldsymbol{\sigma}^{u}+\boldsymbol{\sigma}^{t}\right):\left(\varepsilon^{t}-\boldsymbol{\varepsilon}^{u}\right)+\left(\psi^{u}-\psi^{t}\right)+C \lambda^{t}\left(\sum_{\alpha} q^{\alpha, u}-\sum_{\alpha} q^{\alpha, t}\right)
$$

While many studies (Abdolvand et al., 2012; Juan et al., 2014; Kalidindi, 2001; Proust et al., 2009) assume the driving force for twinning to be the shear stress acting on the twinning system (i.e. $\boldsymbol{\sigma}^{u}: \boldsymbol{\varepsilon}^{t w, t}$ ), a different expression, which is based on thermodynamical arguments, is proposed here. Indeed, according to the above expression, the driving force does not solely depend on the stress state within the untwinned domain. It also involves (1) the stress state within

$\overline{2}$ Though no application is presented here, detwinning, which would correspond to a reduction of the twinned domain, can also be investigated with the proposed constitutive model. 
the twinned domain, (2) the bulk free energy density difference between the twinned and untwinned domains and (3) the contribution associated with the twin interface. Twinning is therefore a favored deformation mode when a reduction of the total free energy is permitted by the growth of the twinned domain.

The resistance to the expansion of the twinned domain is represented by the driving force $g^{t}$ whose expression is:

$$
g^{t}=W \lambda^{t}+C\left(\phi^{t} \sum_{\alpha} q^{\alpha, t}+\left(1-\phi^{t}\right) \sum_{\alpha} q^{\alpha, u}\right)
$$

\subsubsection{Evolution laws}

For the description of the constitutive model to be complete, one must define the evolution laws of the internal variables. For each slip system, the evolution of the plastic shear strain is given by the following viscoplastic flow rule, which takes the form of a power law (Méric and Cailletaud, 1991):

$$
\begin{aligned}
& \dot{\gamma}^{\alpha, u}=\left(\frac{\left\langle\left|\tau^{\alpha, u}\right|-r^{\alpha, u}-R^{\alpha}\right\rangle}{K^{\alpha}}\right)^{N^{\alpha}} \operatorname{sign}\left(\tau^{\alpha, u}\right) \\
& \dot{\gamma}^{\alpha, t}=\left(\frac{\left\langle\left|\tau^{\alpha, t}\right|-r^{\alpha, t}-R^{\alpha}\right\rangle}{K^{\alpha}}\right)^{N^{\alpha}} \operatorname{sign}\left(\tau^{\alpha, t}\right)
\end{aligned}
$$

where $K^{\alpha}$ is a viscosity parameter, $N^{\alpha}$ is a strain rate sensitivity parameter and $R^{\alpha}$ corresponds to the initial value of the critical shear stress. Both $K^{\alpha}$ and $R^{\alpha}$ represent some kind of resistance to plastic slip. However, while $K^{\alpha}$ is associated with lattice friction, $R^{\alpha}$ stands for the resistance opposed by crystallographic defects to plastic slip (e.g. dislocations). $R^{\alpha}$ can thus be assimilated to be the initial value of $r^{t, \alpha}$ and $r^{u, \alpha}$.

Though more sophisticated formulations can be employed, simple isotropic hardening rules with saturation are used here. Such hardening rules are obtained with the following evolution laws for $q^{\alpha, u}$ and $q^{\alpha, t}$ :

$$
\begin{aligned}
& \dot{q}^{\alpha, u}=\left(1-A q^{\alpha, u}\right)\left|\dot{\gamma}^{\alpha, u}\right| \\
& \dot{q}^{\alpha, t}=\left(1-A q^{\alpha, t}\right)\left|\dot{\gamma}^{\alpha, t}\right|
\end{aligned}
$$

where $A$ is a material parameter.

For the evolution of the internal variables associated with the twinning deformation mode $\left(\phi^{t}\right.$ and $\left.\lambda^{t}\right)$, a quite similar description is used. More specifically, the twin volume fraction rate $\dot{\phi}^{t}$ is expressed as a function of the corresponding 
driving force $f^{t}$ according to:

$$
\dot{\phi}^{t}=\left(\frac{\left\langle f^{t}-g^{t}-G^{t}\right\rangle}{L^{t}}\right)^{M^{t}}\left(1-\phi^{t}\right)
$$

where $L^{t}$ is a viscosity parameter, $M^{t}$ is a strain rate sensitivity parameter and $G^{t}$ corresponds to the initial value of the critical driving force for twinning. With the above evolution law, the twin volume fraction is necessarily comprised between zero and one. For the evolution of the hardening variable $\lambda^{t}$, the following non-linear evolution equation is employed:

$$
\dot{\lambda}^{t}=\left(1-B \lambda^{t}\right) \dot{\phi}^{t}
$$

where $B$ is a material parameter controlling the asymptotic value of $\lambda^{t}$.

\subsubsection{Nucleation criterion}

At the beginning of a deformation process, the volume element $v$ is usually homogeneous with a zero twin volume fraction. It is therefore necessary to have a nucleation criterion to determine whether a twinned domain should be created or not. Also, the criterion must allow for selection of the most appropriate twinning system amongst all the possible variants. In the present work, the driving force for nucleation is assumed to be the same as for growth. When no twinned domain exists, the driving force $f^{t}$ is thus calculated at each time $t$ for all the possible twinning systems. The nucleation event takes place when the driving force $f^{t}$ reaches the critical value $g^{t}+G^{t}$. When this nucleation condition is met, the crystallographic orientation of the newly formed twinned domain is determined according to the orientation relationship of the selected twinning system.

When a nucleation event occurs, no inheritance mechanism is considered: the plastic shear strains $\gamma^{\alpha, t}$ and the isotropic variables $q^{\alpha, t}$ associated with the slip systems of the newly created twinned domain are initially fixed to zero. As a result, the plastic strain and rotation tensors for the twinned domain are initially equal to the twinning strain and rotation tensors (i.e. $\varepsilon^{p, t}=\varepsilon^{t w, t}$ and $\left.\boldsymbol{\omega}^{p, t}=\boldsymbol{\omega}^{t w, t}\right)$. The partition method described in section 2.1.2 is then used to determine the initial strain, rotation and stress tensors associated with the newly created twinned domain.

Also, in the present formulation, once a nucleation event occurs, no further nucleation event is allowed. Indeed, though multiple twinned domains could be considered with the partition method described in 2.1.2, the corresponding twin interfaces would have to be parallel. Since this assumption regarding the orientation of twin interfaces is not realistic, the coexistence of multiple twinned domains in a volume element is not allowed here. This is however 
expected to be a minor limitation since, if the volume element is small enough, the probability of having two (or more) twins is quite low.

\subsection{Spectral method}

Let us now consider a volume element with volume $V$ and external boundary $\partial V$. The volume element $V$ is representative of a polycrystalline aggregate consisting of a collection of crystallites being perfectly bonded across their interfaces. For a given loading path, the overall behavior of the representative volume element is given by the evolution of the macroscopic stress tensor $\boldsymbol{\Sigma}(t)$ as a function of the prescribed macroscopic strain and rotation tensors $\boldsymbol{E}(t)$ and $\Omega(t)$.

For the application of the FFT spectral method, the representative volume element is divided into $N_{1} \times N_{2} \times N_{3}$ voxels. The stress, strain and rotation tensors associated with a voxel of volume $v(\overline{\boldsymbol{x}}, t)$ occupying a barycentric position $\overline{\boldsymbol{x}}$ are denoted by $\overline{\boldsymbol{\sigma}}(\overline{\boldsymbol{x}}, t), \overline{\boldsymbol{\varepsilon}}(\overline{\boldsymbol{x}}, t)$ and $\overline{\boldsymbol{\omega}}(\overline{\boldsymbol{x}}, t)$. For each voxel, the mechanical response is determined by the constitutive model proposed in the previous section which provides a relation between the stress and strain tensors $\overline{\boldsymbol{\sigma}}(\overline{\boldsymbol{x}}, t)$ and $\overline{\boldsymbol{\varepsilon}}(\overline{\boldsymbol{x}}, t)$. The integration of constitutive relations is performed with a semi-implicit finite difference method. This method involves a non-linear set of equations which is numerically solved with a Newton-Raphson method.

In order to connect the local strain and rotation tensors $\overline{\boldsymbol{\varepsilon}}(\overline{\boldsymbol{x}}, t)$ and $\overline{\boldsymbol{\omega}}(\overline{\boldsymbol{x}}, t)$ to the prescribed macroscopic strain and rotation tensors $\boldsymbol{E}(t)$ and $\boldsymbol{\Omega}(t)$, the spectral method proposed by Moulinec and Suquet (1998) and Müller (1996) is employed. Though it has been originally developed for composite materials, this method is now widely used to investigate the mechanical behavior of polycrystalline materials with an elastic-viscoplastic behavior (Grennerat et al., 2012; Lebensohn et al., 2012; Robert and Mareau, 2015; Shanthraj et al., 2015). Assuming the representative volume element to be subjected to periodic boundary conditions and introducing a homogeneous reference elastic medium with stiffness tensor $\boldsymbol{C}$, the spectral method aims at finding a numerical solution to the following integral equations:

$$
\begin{aligned}
\overline{\boldsymbol{\varepsilon}}(\overline{\boldsymbol{x}}, t) & =\boldsymbol{E}(t)-\int_{V} \boldsymbol{\Gamma}(\overline{\boldsymbol{x}}-\overline{\boldsymbol{y}}): \delta \overline{\boldsymbol{\sigma}}(\overline{\boldsymbol{y}}, t) d V \\
\overline{\boldsymbol{\omega}}(\overline{\boldsymbol{x}}, t) & =\boldsymbol{\Omega}(t)-\int_{V} \boldsymbol{\Pi}(\overline{\boldsymbol{x}}-\overline{\boldsymbol{y}}): \delta \overline{\boldsymbol{\sigma}}(\overline{\boldsymbol{y}}, t) d V
\end{aligned}
$$

where $\boldsymbol{\Gamma}$ and $\boldsymbol{\Pi}$ are respectively the symmetric and skew-symmetric parts of the Green tensor associated with the homogeneous elastic reference medium and $\delta \overline{\boldsymbol{\sigma}}(\overline{\boldsymbol{x}}, t)$ is the polarization tensor field:

$$
\delta \overline{\boldsymbol{\sigma}}(\overline{\boldsymbol{x}}, t)=\overline{\boldsymbol{\sigma}}(\overline{\boldsymbol{x}}, t)-\boldsymbol{C}: \overline{\boldsymbol{\varepsilon}}(\overline{\boldsymbol{x}}, t)
$$


The application of the Fourier transform, which is denoted by a' ' ' symbol, to equations (55) and (56) allows for transforming the circular convolution operations into tensor products:

$$
\begin{aligned}
& \hat{\bar{\varepsilon}}(\boldsymbol{\xi}, t)= \begin{cases}\boldsymbol{E}(t), & \boldsymbol{\xi}=\mathbf{0} \\
-\hat{\boldsymbol{\Gamma}}(\boldsymbol{\xi}): \delta \hat{\overline{\boldsymbol{\sigma}}}(\boldsymbol{\xi}, t), & \boldsymbol{\xi} \neq \mathbf{0}\end{cases} \\
& \hat{\overline{\boldsymbol{\omega}}}(\boldsymbol{\xi}, t)= \begin{cases}\boldsymbol{\Omega}(t), & \boldsymbol{\xi}=\mathbf{0} \\
-\hat{\boldsymbol{\Pi}}(\boldsymbol{\xi}): \delta \hat{\overline{\boldsymbol{\sigma}}}(\boldsymbol{\xi}, t), & \boldsymbol{\xi} \neq \mathbf{0}\end{cases}
\end{aligned}
$$

where $\boldsymbol{\xi}$ is the frequency vector. According to the spectral method, the integral equations are conveniently solved in the frequency domain and an inverse transformation is then applied to express the local strain and rotation tensors in the original spatial domain. However, because the local polarization tensor $\delta \overline{\boldsymbol{\sigma}}(\overline{\boldsymbol{x}}, t)$ depends on the local strain tensor $\overline{\boldsymbol{\varepsilon}}(\overline{\boldsymbol{x}}, t)$, an iterative resolution procedure is required to find the strain tensor. In the present work, the procedure proposed by Eyre and Milton (1999) is used.

\section{Application to a AZ31 magnesium alloy}

The model presented in the previous section provides a way of estimating both the local and macroscopic stress states in a representative volume element subjected to known loading conditions. It is thus well suited for the study of the development of texture and internal strains in metallic alloys since it allows for quantifying of the relative importance of the different possible plastic deformation modes. In this section, the proposed model is used to investigate the mechanical behavior of a AZ31 magnesium alloy for which an extensive experimental dataset has been obtained by Clausen et al. (2008).

\subsection{Experimental data}

Clausen et al. (2008) carried out neutron diffraction measurements during the mechanical testing of a magnesium alloy cylindrical sample. As shown in figure 2, the magnesium alloy is initially characterized by an extrusion texture where most of the basal poles are oriented perpendicularly to the extrusion direction. A compressive load was applied to the sample along the cylinder axis, in the direction where almost all the grains are favorably oriented for tensile twinning. The evolution of the macroscopic stress as a function of the macroscopic strain is represented in figure 3. Also, during the compression test, lattice strains were measured for the $\{10 \overline{1} 0\},\{0002\},\{10 \overline{1} 1\}$ and $\{11 \overline{2} 0\}$ diffraction peaks in two directions: axial (parallel to the loading axis) and 


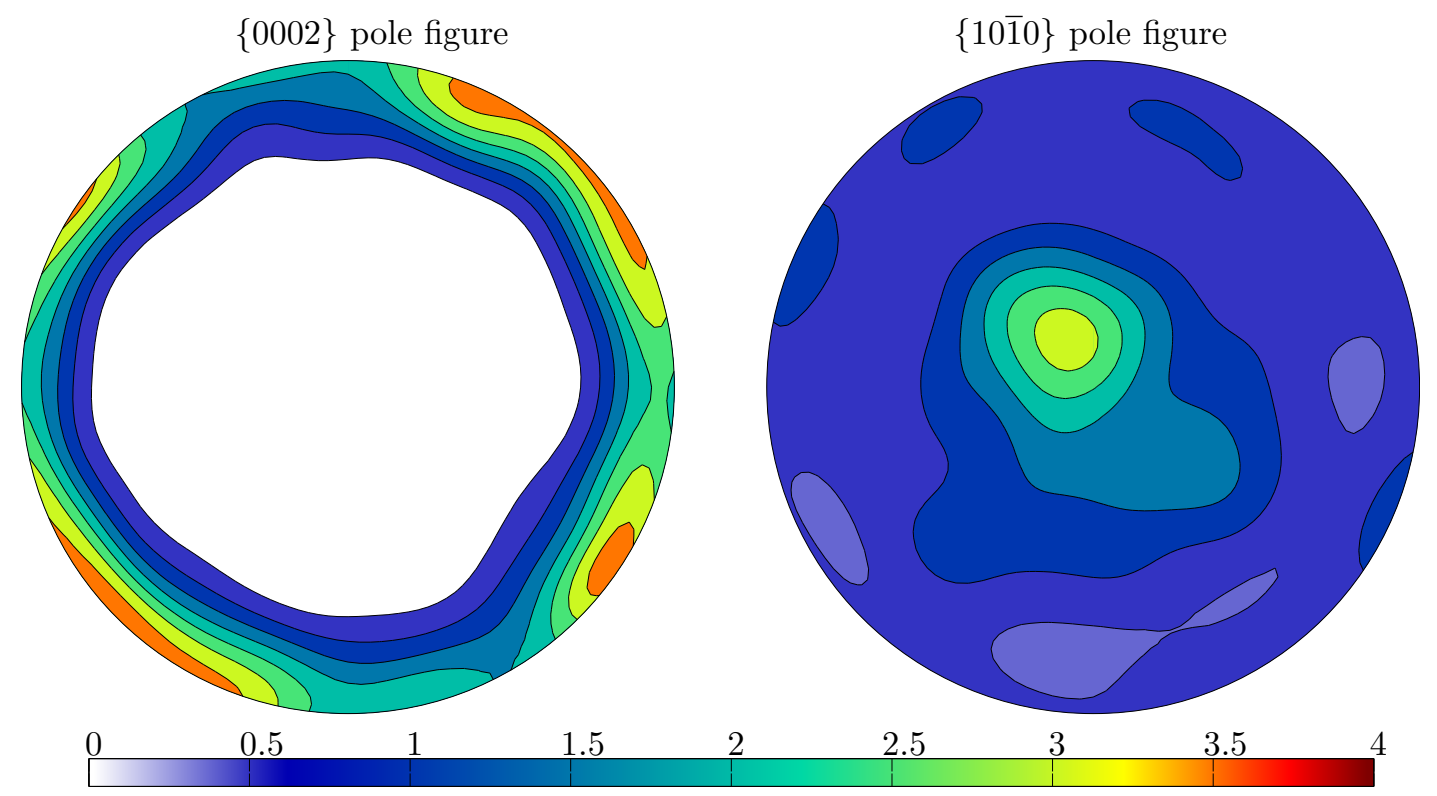

Figure 2. Initial pole figures for the AZ31 magnesium alloy. The pole figure centers correspond to the original rod extrusion direction, which is also the axial loading direction. Pole figures were plotted using the MTEX package (Hielscher and Schaeben, 2008).

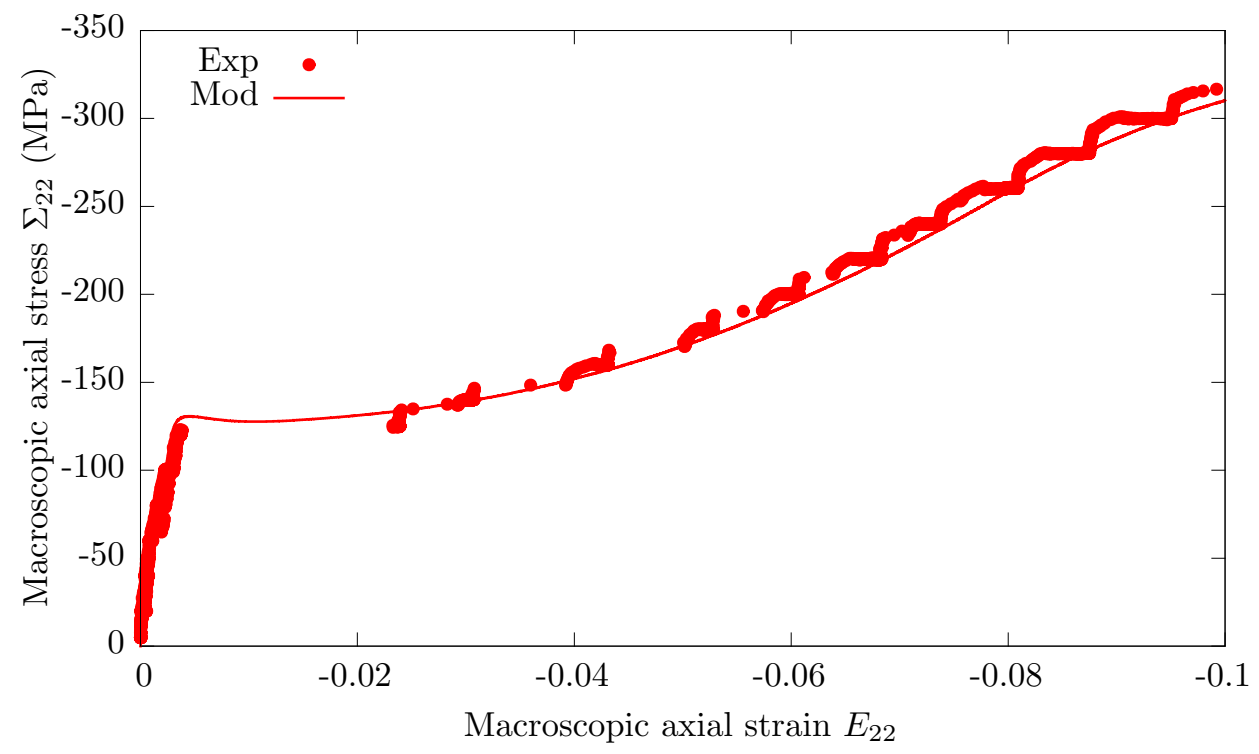

Figure 3. Comparison between the experimental (Exp) and calculated (Mod) stress-strain curves obtained for the compression test conducted along the axial direction of the AZ31 magnesium alloy cylindrical specimen.

radial (perpendicular to the loading axis). The evolution of the lattice strains are plotted in figures 4 and 5 for these two directions. In addition, based on diffraction peak intensities, the twin volume fraction was evaluated during the test. The twin volume fraction is represented as a function of the macroscopic axial strain in figure 6 . 


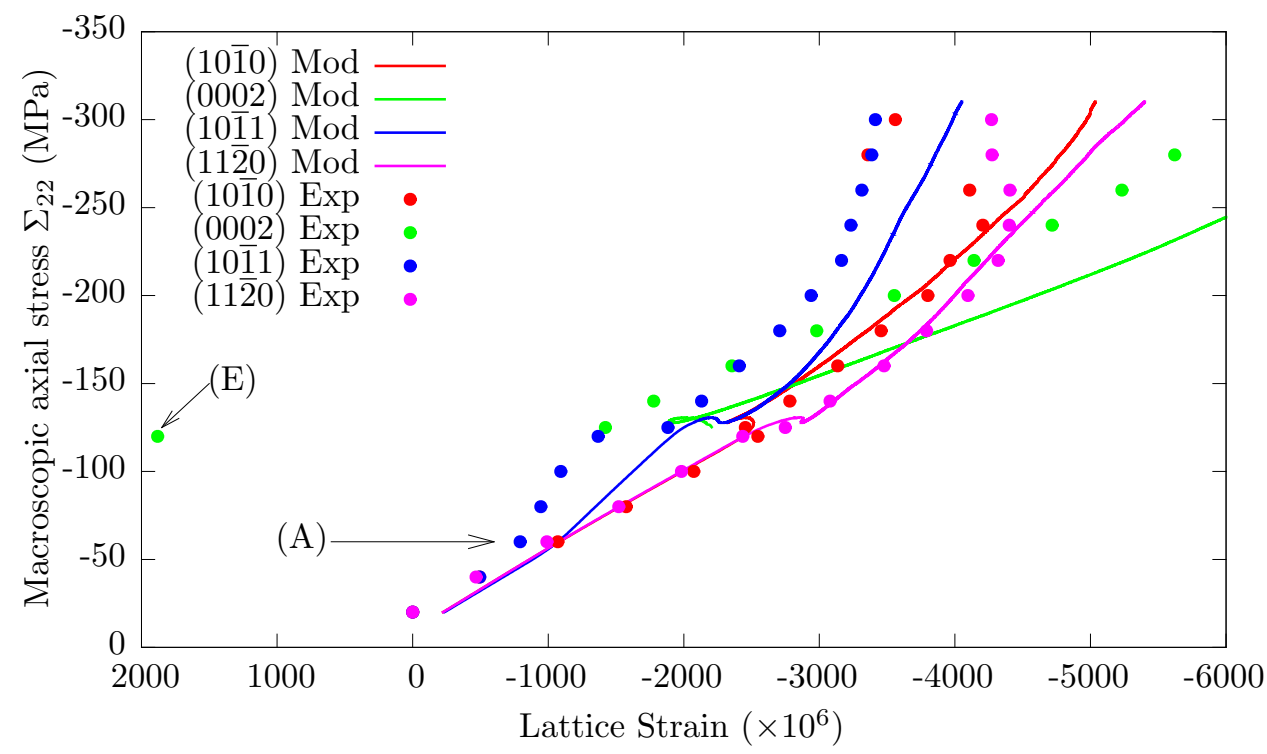

Figure 4. Comparison between the experimental (Exp) and calculated (Mod) axial lattice strains obtained for different crystallographic planes during the compression test conducted along the axial direction of the AZ31 magnesium alloy cylindrical specimen.

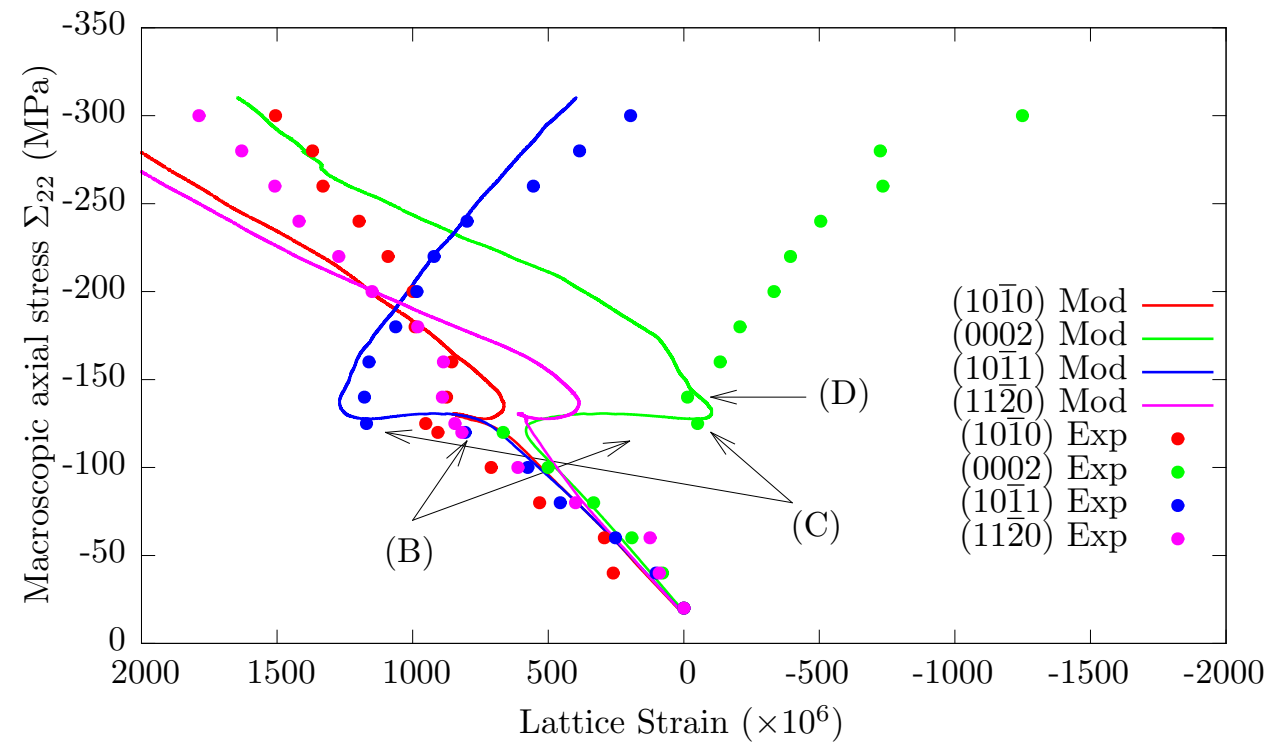

Figure 5. Comparison between the experimental (Exp) and calculated (Mod) radial lattice strains obtained for different crystallographic planes during the compression test conducted along the axial direction of the AZ31 magnesium alloy cylindrical specimen.

\subsection{Material parameters}

The calculations were performed with a polycrystal consisting of 500 equiaxed grains that was constructed from a periodic Voronoï tessellation according to 


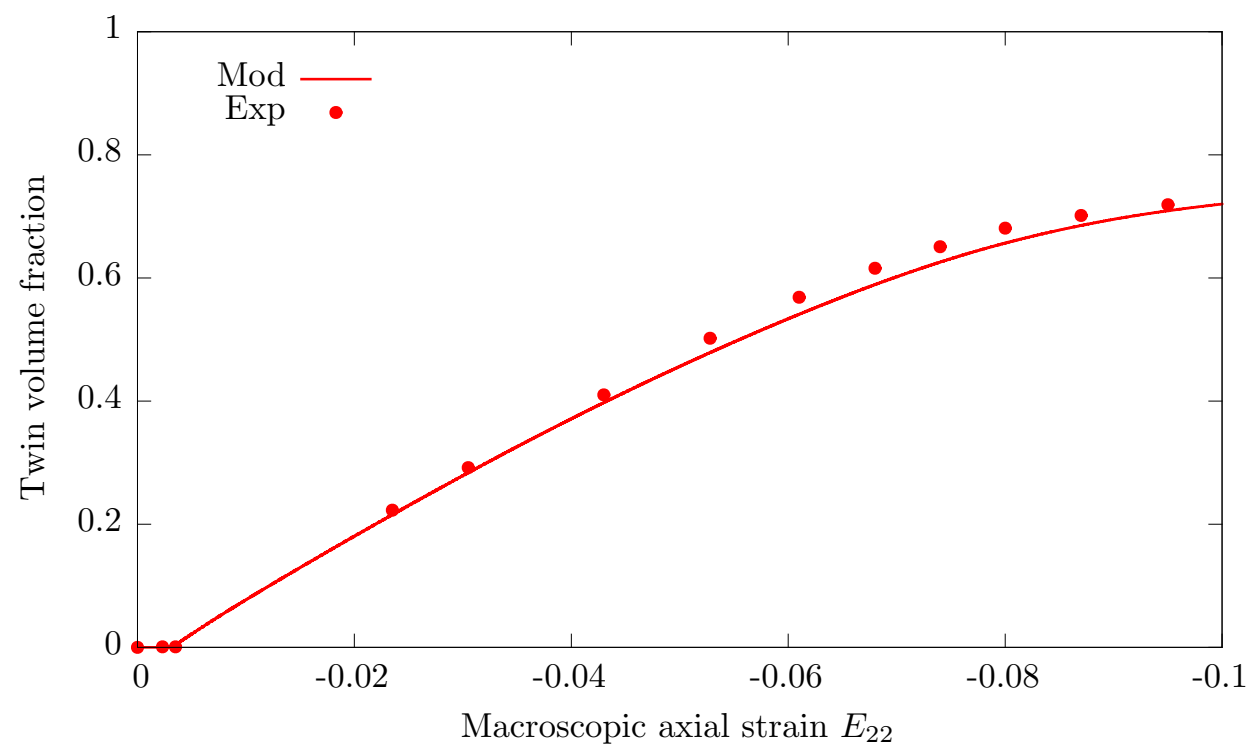

Figure 6. Comparison between the experimental (Exp) and calculated (Mod) twin volume fraction evolutions obtained during the compression test conducted along the axial direction of the AZ31 magnesium alloy cylindrical specimen.

the procedure of Fritzen et al. (2009). For the application of the spectral method, the polycrystal was then discretized into $128 \times 128 \times 128$ voxels. The crystallographic orientations were extracted from measured pole figures to ensure that the volume element is representative of the real material. The plastic deformation modes that are considered are the same as in (Agnew et al., 2006; Clausen et al., 2008): the plastic activity is restricted to the basal (Bas), first order prismatic (Pri) and second order pyramidal (Pyr) slip systems while only tensile twinning (TTw) is accounted for.

Except for the single crystal elastic constants, which are those identified for pure magnesium by Simmons and Wang (1971), the material parameters were adjusted to best reproduce the macroscopic stress-strain behavior, the twin volume fraction evolution and the lattice strain development. The material parameters are listed in table 1 . An important issue concerns the unicity of the set of material parameters. Indeed, with the considered experimental dataset, the optimal set of material parameters is not necessarily unique. As a result, there are some material parameters, especially the hardening matrix $H$, for which some additional experimental data would be needed to obtain more accurate values.

\subsection{Discussion}

As shown in figure 3, the macroscopic behavior, which exhibits a two-stage hardening, is correctly described by the model. The plateau observed in the 


\begin{tabular}{|c|c|c|c|c|}
\hline \multicolumn{5}{|c|}{ Elasticity } \\
\hline$C_{11}(\mathrm{GPa})$ & $C_{12}(\mathrm{GPa})$ & $C_{13}(\mathrm{GPa})$ & $C_{33}(\mathrm{GPa})$ & $C_{44}(\mathrm{GPa})$ \\
\hline 59.75 & 23.24 & 21.17 & 61.70 & 16.39 \\
\hline \multicolumn{5}{|c|}{ Basal slip systems } \\
\hline$R^{\alpha}(\mathrm{MPa})$ & $K^{\alpha}(\mathrm{MPa})$ & $N^{\alpha}$ & $H^{\alpha \alpha}$ & $H^{\alpha \beta}$ \\
\hline 10 & 10 & 50 & 1.1 & 1.0 \\
\hline \multicolumn{5}{|c|}{ First order prismatic slip systems } \\
\hline$R^{\alpha}(\mathrm{MPa})$ & $K^{\alpha}(\mathrm{MPa})$ & $N^{\alpha}$ & $H^{\alpha \alpha}$ & $H^{\alpha \beta}$ \\
\hline 50 & 60 & 50 & 1.1 & 1.0 \\
\hline \multicolumn{5}{|c|}{ Second order pyramidal slip system } \\
\hline$R^{\alpha}(\mathrm{MPa})$ & $K^{\alpha}(\mathrm{MPa})$ & $N^{\alpha}$ & $H^{\alpha \alpha}$ & $H^{\alpha \beta}$ \\
\hline 140 & 80 & 50 & 1.1 & 1.0 \\
\hline \multicolumn{5}{|c|}{ Twinning } \\
\hline$G^{t}(\mathrm{MPa})$ & $L^{t}(\mathrm{MPa})$ & $M^{t}$ & & \\
\hline 5.5 & 4 & 50 & & \\
\hline \multicolumn{5}{|c|}{ Hardening } \\
\hline$Q(\mathrm{MPa})$ & $A$ & $W(\mathrm{MPa})$ & $B$ & $C(\mathrm{MPa})$ \\
\hline 10 & 0 & -50 & 10 & 30 \\
\hline
\end{tabular}

Table 1

Material parameters for the AZ31 magnesium alloy. Only independent elastic constants are indicated.

first stage is mostly due to the development of an important twinning activity. Indeed, the activities of the different deformation modes, which are represented in figure 7 , show that the nucleation of tensile twins coincides with the initial yielding. Also, although basal slip is the first deformation mode to be active, it provides a negligible contribution to the macroscopic strain. The second stage, which is usually attributed to the multiplication of twin boundaries (Basinski et al., 1997), is captured because a coupling between slip and twinning modes is considered in the constitutive model. Indeed, when interactions between twin boundaries and slip systems are ignored (i.e. $C=0 \mathrm{MPa}$ ), the model fails in correctly reproducing the experimentally observed hardening behavior. For this magnesium alloy, the role of twin boundaries, which act as barriers to dislocation motion, is thus important for explaining the mechanical behavior.

The calculated and measured evolution of lattice strains are compared to each other in figures 4 and 5. For most planes, the evolution of lattice strains is generally well described by the model. As shown in figure 4, the inflexion ob- 


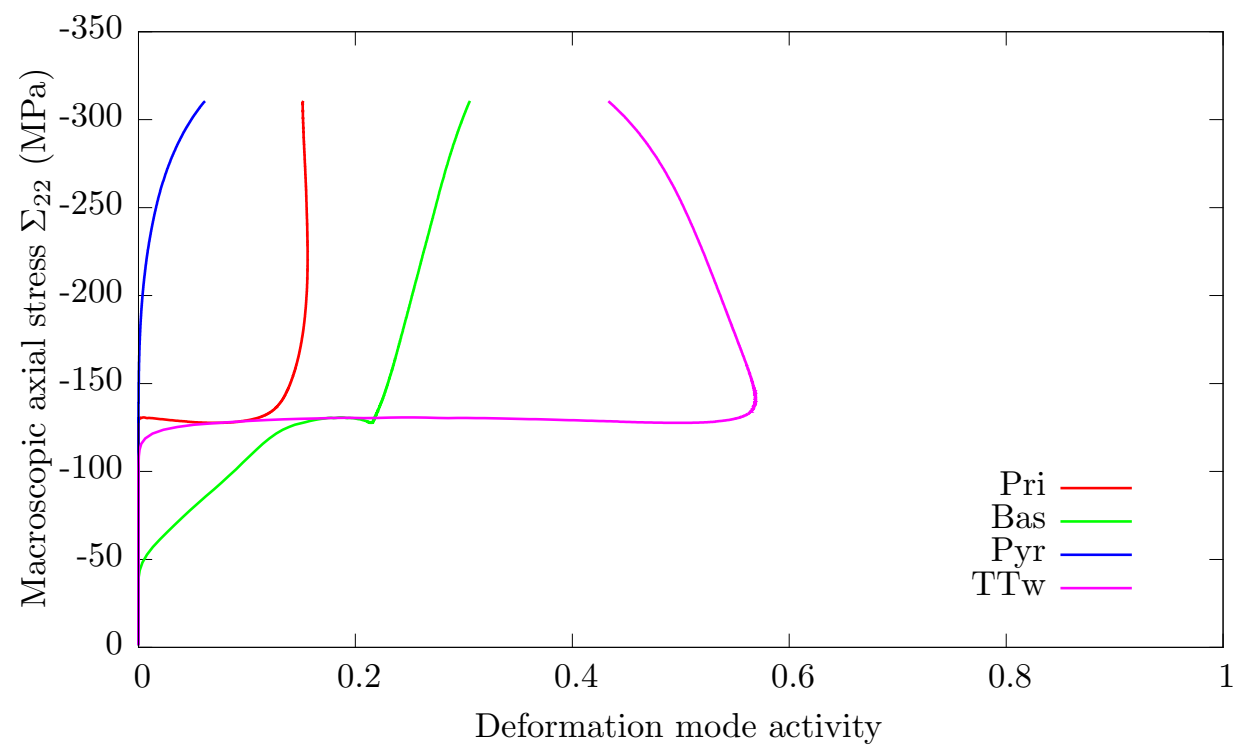

Figure 7. Calculated activities of the different deformation modes during the compression test conducted along the axial direction of the AZ31 magnesium alloy cylindrical specimen. For a given deformation mode, the activity is defined as the ratio between the average equivalent plastic strain produced by this mode and the equivalent total macroscopic strain.

served for the $\{10 \overline{1} 0\}$ plane in the axial direction at $-60 \mathrm{MPa}(\mathrm{A})$ is caused by the early activity of the basal slip mode. Interestingly, in contrast with other modeling attempts, the discontinuities observed for the $\{0002\}$ and $\{10 \overline{1} 1\}$ planes in the radial direction for an applied stress of -120 MPa (B) are well reproduced by the model. Such discontinuities are caused by twinning which is responsible for a significant redistribution of internal stresses. These results thus demonstrate the importance of an appropriate strategy for partitioning the stress and strain fields between the twinned and untwinned domains. According to the model, the slope reversals for the $\{0002\}$ and $\{10 \overline{1} 1\}$ lattice strains in the radial direction (C) are due to the activation of prismatic slip systems. The present model however fails in reproducing the inflexion about $-140 \mathrm{MPa}$ for the $\{0002\}$ lattice strain in the radial direction (D). This discrepancy between numerical and experimental results could be explained by the fact that secondary twinning is not considered in the proposed model and/or an overestimation of the hardening contribution associated with the interactions between slip systems and twin planes. Carrying out modelling with a larger number of grains might also assist in obtaining an accurate description of internal strains, which are more sensitive than texture or macroscopic stressstrain response to the statistical representation, particularly in the direction perpendicular to the applied load (Oliver et al., 2004).

Also, while the growth of a twinned domain is properly depicted by the proposed constitutive model, the estimation of the initial stress state of twinned domains still remains to be improved. This aspect of the nucleation process is 
underlined by observing the evolution of the $\{0002\}$ lattice strain in the axial direction. Indeed, because of the initial crystallographic texture, the $\{0002\}$ reflection is absent in the axial direction at the beginning of the compression test. In this direction, the $\{0002\}$ diffraction peak is observed only when the twin volume fraction reaches a significant value. The initial value of the $\{0002\}$ lattice strain therefore provides some information regarding the initial stress state within twinned domains. As illustrated by figure 5, though the evolution of the $\{0002\}$ lattice strain is reasonably well described, the model fails in estimating the initial value (E) which, according to diffraction measurements, is far into tension (about 2000 microstrains). Recent 3DXRD data suggests this initial twin strain may vary considerably in value depending on local conditions (Abdolvand et al., 2015).

The evolution of the twin volume fraction is shown in figure 6 . The calculated evolution is in good agreement with the measurement: the rapid increase due to the activation of the twinning deformation mode and the saturation observed towards the end of the test are both well reproduced. Also, as shown in figure 8, during the compression test, the local twin volume fraction progressively increases to form the characteristic intragranular lamellar structure that is often observed for twinned grains (Capolungo et al., 2009b). With an increasing applied strain, both the number of twins and the thickness of the lamellar structure tend to increase. At the end of the compression test, most grains are entirely filled with tensile twins. To describe how the morphological texture is impacted by twinning, the localized aspect of twin propagation must be accounted for. To include this aspect in the present study, a softening mechanism is considered by giving a negative value to the parameter $W$. This corresponds to the situation where an increase of the twin volume fraction is accompanied by a reduction of the resistance to the expansion of the twinned domain. This softening mechanism, which is explained by the fact that nucleation is expected to require a larger driving force than propagation, favors a localized (rather than diffuse) development of twinned regions. Also, while the morphological texture evolution is generally well depicted, some aspects need to be improved. For instance, there are some intragranular regions where the twinned microstructure is not accurately described since the local twin volume fraction is possibly inferior to $100 \%$ inside twin lamellas (see for instance figure 6c). Indeed, because twinning is modelled as a continuous temporal process, the twinned microstructure develops in a smooth, rather than abrupt, manner.

The final basal and prismatic pole figures are plotted in figure 9. The strong texture change induced by tensile twinning that was experimentally observed by Clausen et al. (2008) is correctly reproduced by the model: the density of basal poles decreases along the rim and increases at the center while the inverse is observed for prismatic poles. Therefore, although no specific criterion was developed for nucleation, the model provides a reasonable description of the twin variant selection phenomenon. To further investigate the variant selection 
(a)

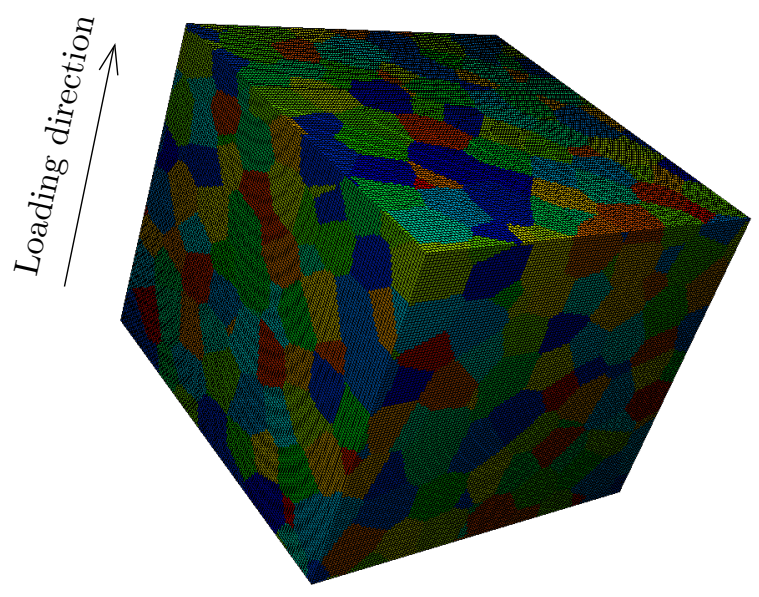

(c)

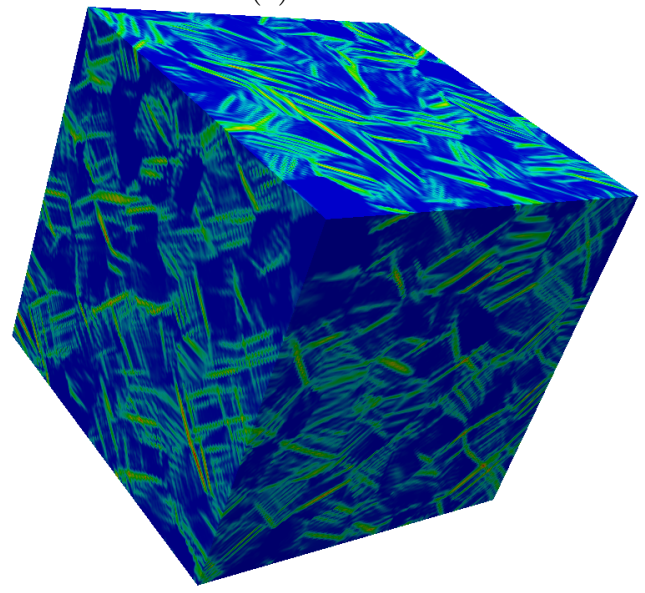

(e)

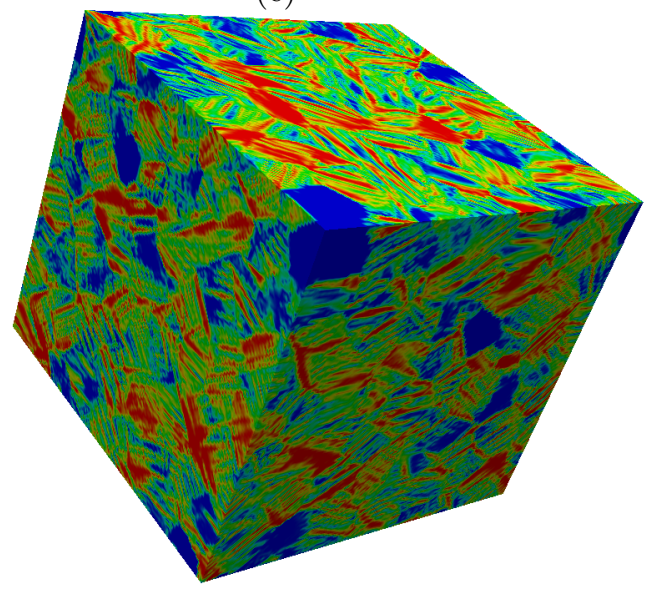

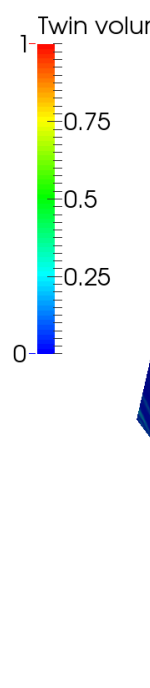

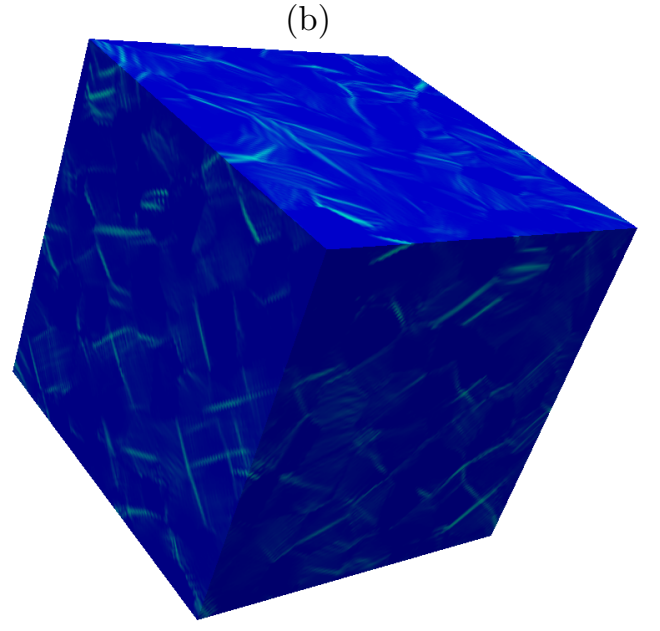

(d)

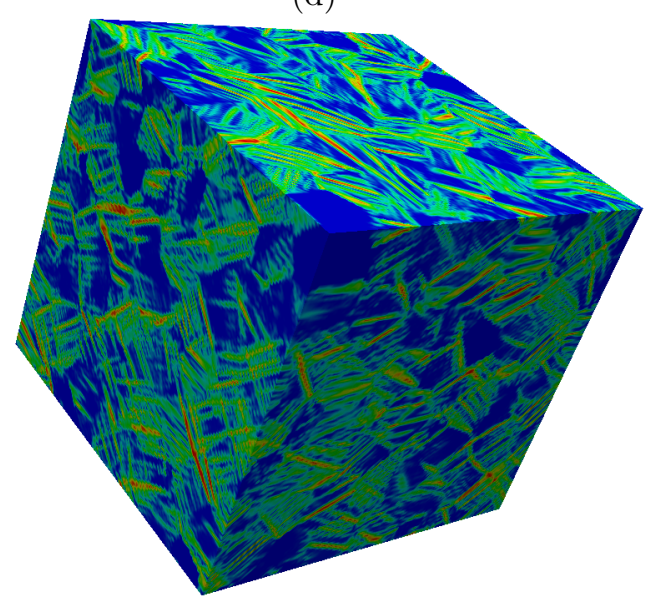

(f)

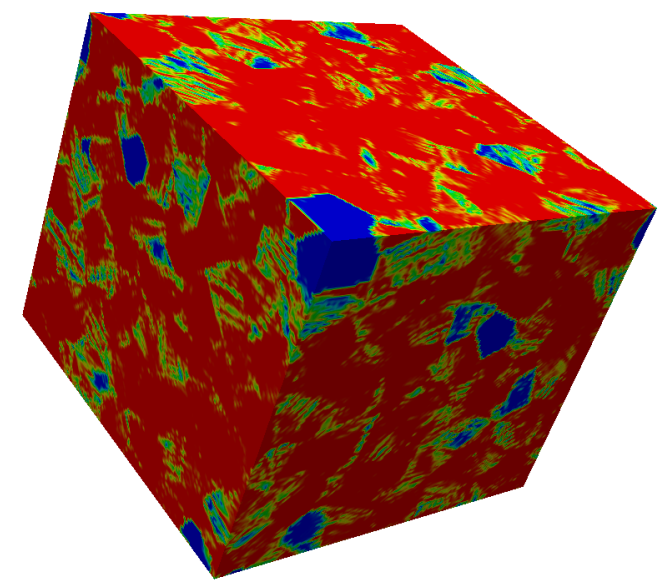

Figure 8. Representative volume element after discretization into $128 \times 128 \times 128$ voxels (a). Evolution of the local twin volume fraction for different imposed axial strain: $E_{22}=-0,5 \%$ (b), $E_{22}=-1 \%$ (c), $E_{22}=-2 \%$ (d), $E_{22}=-5 \%$ (e) and $E_{22}=-10 \%$ (f). 


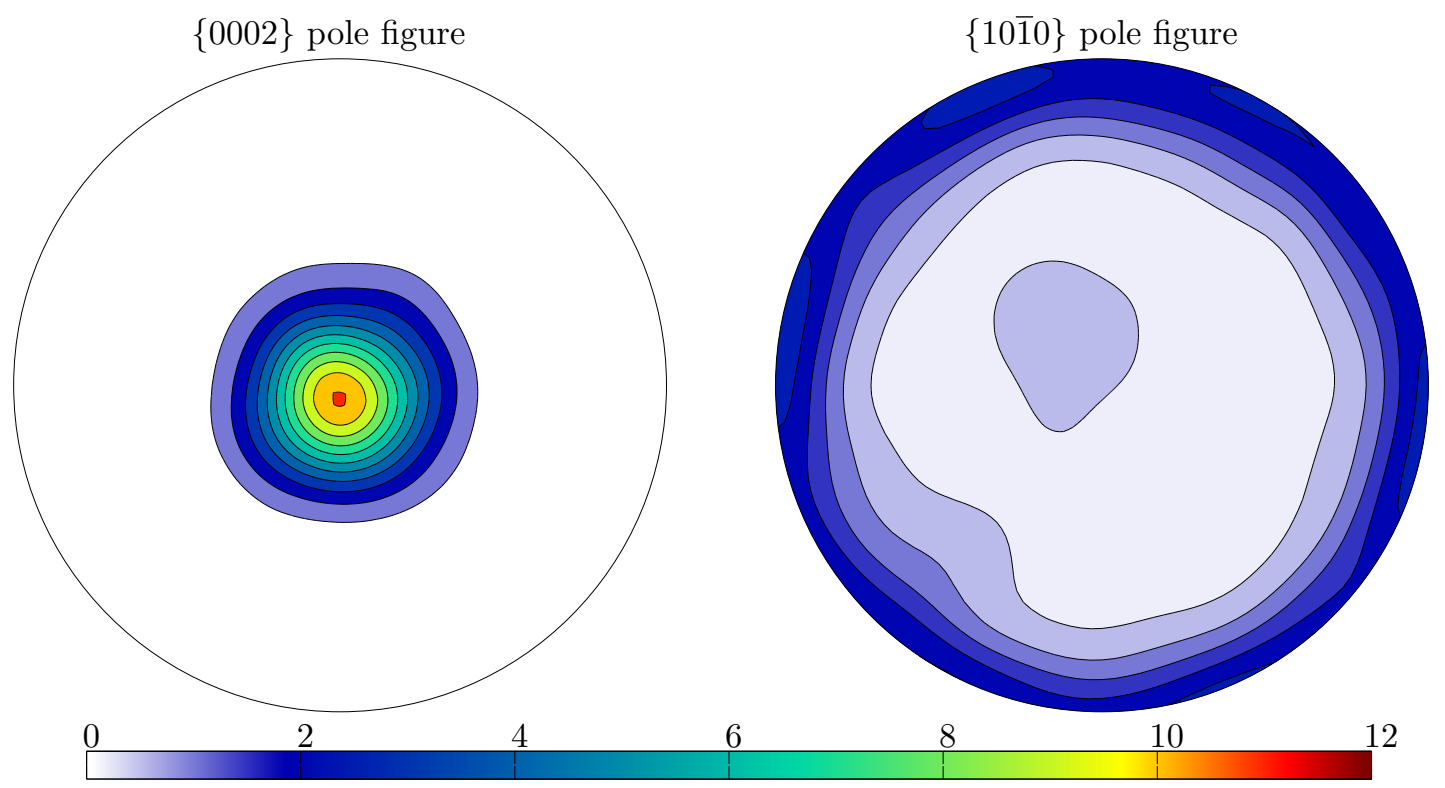

Figure 9. Calculated final pole figures for the AZ31 magnesium alloy after the compression test conducted along the axial direction. The pole figure centers correspond to the original rod extrusion direction, which is also the axial loading direction. Pole figures were plotted using the MTEX package (Hielscher and Schaeben, 2008).

process, the total twin volume fraction at the end of the compression test is plotted as a function of the Schmid factor in figure 10. Since the volume average Schmid factor is quite high (about 0.44), the twin variant selection process is strongly governed by the macroscopic loading direction. Thus, though efforts were made to account for local stress field fluctuations, these fluctuations seem to play a secondary role on the twin variant selection process in this case.

\section{Conclusions}

In this work, a constitutive model accounting for the contribution of deformation twinning is proposed. The development of the model is based on a micromechanical approach that describes the interactions between twinned and untwinned domains in an explicit manner. Following thermodynamical arguments, an alternative expression for the driving force for twinning is obtained. For a given twinning system, the driving force depends not only on the corresponding shear stress but also on the different state variables. Finally, to account for the polycrystalline nature of metallic materials, the constitutive relations are implemented in a FFT spectral solver.

To demonstrate the relevance of the proposed model, the behavior of a AZ31 magnesium alloy subjected to uniaxial compression is then investigated. The experimental results of Clausen et al. (2008) are used to identify material 


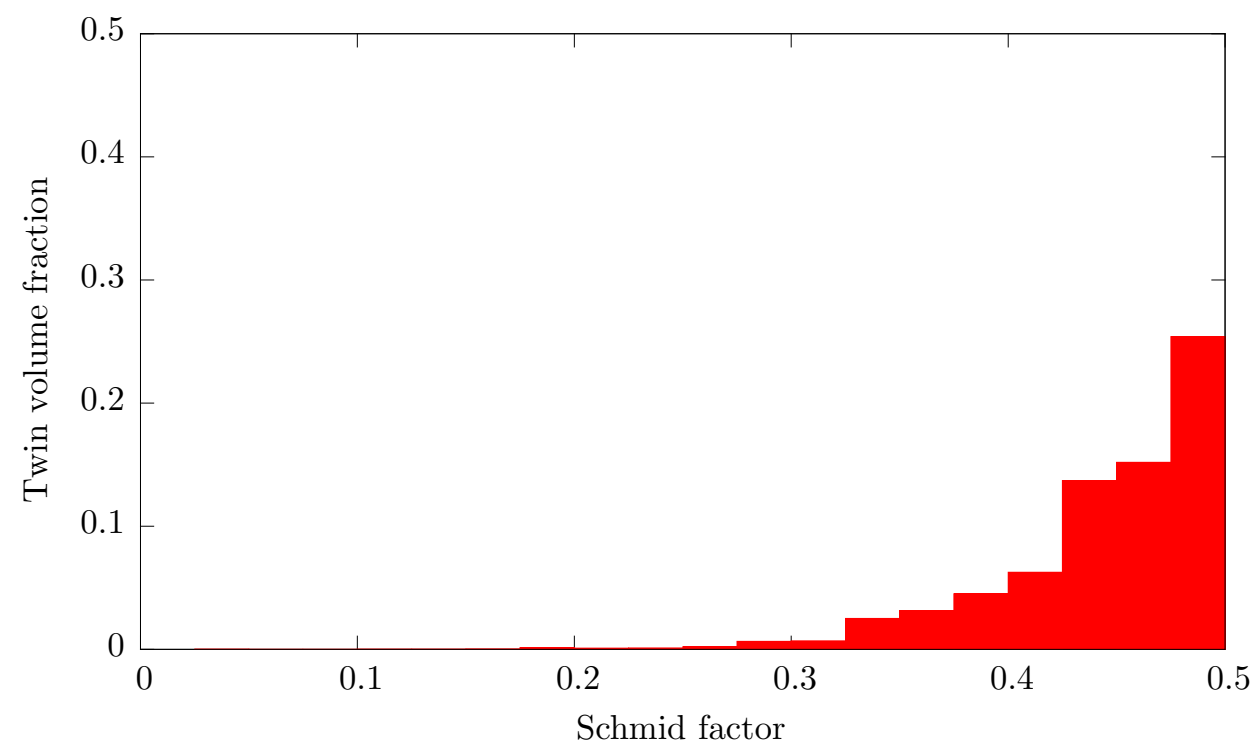

Figure 10. Distribution of the Schmid factor associated with the twins observed at the end of the compression test (i.e. $E_{22}=-10 \%$ ) conducted along the axial direction of the AZ31 magnesium alloy cylindrical specimen. The volume average schmid factor is about 0.44 .

parameters. Since the interactions between twinned and untwinned domains are explicitly considered, a reasonable description of the evolution of lattice strains is obtained. Also, the model correctly reproduces the characteristic intragranular lamellar structure that is classically observed for twinned grains. The observation of texture evolutions shows that the reorientation process associated with twinning is properly described with the proposed model.

Future work should focus on the description of the nucleation stage since the present model fails to correctly estimate the initial stress state within newly formed twinned domains. In this perspective, the recent development of $3 \mathrm{D} \mathrm{X}$ ray diffraction techniques (Abdolvand et al., 2015; Aydiner et al., 2009; Bieler et al., 2014) is expected to provide some additional information to help in proposing an appropriate strategy for the description of the nucleation event.

\section{Acknowledgements}

The authors wish to thank Bjørn Clausen from LANSCE-LC for providing the experimental dataset for magnesium. This work was financed by $\mathrm{Nu}$-Tech Precision metals, NSERC, COG, and OPG under the Industrial Research Chair program in Nuclear Materials at Queen's University. 


\section{References}

Abdolvand, H., Daymond, M.R., Mareau, C., 2012. Incorporation of twinning into a crystal plasticity finite element model: Evolution of lattice strains and texture in Zircaloy-2. International Journal of Plasticity 27, 1721-1738.

Abdolvand, H., Majkut, M., Oddershede, J., Schmidt, S., Lienert, U., Diak, B.J., Withers, P.J., Daymond, M.R., 2015. On the deformation twinning of Mg AZ31B: A three-dimensional synchrotron X-ray diffraction experiment and crystal plasticity finite element model. International Journal of Plasticity 70, 77-97.

Agnew, S.R., Brown, D.W., Tomé, C.N., 2006. Validating a polycrystal model for the elastoplastic response of magnesium alloy AZ31 using in situ neutron diffraction. Acta Materialia 54, 4841-4852.

Ardeljan, M., McCabe, R.J., Beyerlein, I.J., Knezevic, M., 2015. Explicit incorporation of deformation twins into crystal plasticity finite element models. Comput. Methods Appl. Mech. Engrg. 295, 396-413.

Asaro, R.J., Needleman, A., 1985. Texture development and strain hardening in rate dependent polycrystals. Acta Metall. 33, 923-953.

Aydiner, C.C., Bernier, J.V., Clausen, B., Lienert, U., Tomé, C.N., Brown, D.W, 2009. Evolution of stress in individual grains and twins in a magnesium alloy aggregate. Phys Rev B 80, 024113.

Basinski, Z.S., Szczerba, M.S., Niewczas, M., Embury, J.D., Basinski, S.J., 1997. The transformation of slip dislocations during twinning of copperaluminum alloy crystals. Revue de Metallurgie. Cahiers D'Informations Techniques 94 , 1037-1043.

Bieler, T.R., Wang, L., Beaudoin, A.J., Kenesei, P., Lienert, U., 2014. In situ characterization of twin nucleation in pure Ti using 3D-XRD. Metallurgical and Materials Transactions A: Physical Metallurgy and Materials Science 45, 109-122.

Brown, D.W., Agnew, S.R., Bourke, M.A.M., Holden, T.M., Vogel, S.C., Tomé, C.N., 2005. Internal strain and texture evolution during deformation twinning in magnesium. Materials Science and Engineering A 399, 1-12.

Capolungo, L., Beyerlein, I.J., Kaschner, G.C., Tomé, C.N., 2009. On the interaction between slip dislocations and twins in HCP Zr. Materials Science and Engineering A 513-514, 42-51.

Capolungo, L., Marshall, P.E., McCabe, R.J., Beyerlein, I.J., Tomé, C.N., 2009. Nucleation and growth of twins in Zr: A statistical study. Acta Materialia 57, 6047-6056.

Cheng, J., Ghosh, S., 2015. A crystal plasticity FE model for deformation with twin nucleation in magnesium alloys. International Journal of Plasticity 67, $148-170$.

Cherkaoui, M., 2003. Constitutive equations for twinning and slip in lowstacking-fault-energy metals: a crystal plasticity-type model for moderate strains. Philosophical Magazine 83, 3945-3958.

Clausen, B., Tomé, C.N., Brown, D.W., Agnew, S.R., 2008. Reorientation and 
stress relaxation due to twinning: Modeling and experimental characterization for Mg. Acta Mater. 56, 2456-2468.

Cuitiño, A.M., Ortiz, M., 1992. Computational modelling of single crystals. Modell. Simul. Mater. Sci. Engrg. I, 225-263.

Eyre, D.J., Milton, G.W., 1999. A fast numerical scheme for computing the response of composites using grid renement. EPJ Applied Physics 6, 41-47.

Fritzen, F., Böhlke, T., Schnack, E., 2009. Periodic three-dimensional mesh generation for crystalline aggregates based on Voronoi tessellations. Computational Mechanics 43, 701-713.

Grennerat, F., Montagnat, M., Castelnau, O., Vacher, P., Moulinec, H., Suquet, P., Duval, P., 2012. Experimental characterization of the intragranular strain field in columnar ice during transient creep. Acta Materialia 60, 36553666 .

Hielscher, R., Schaeben, H., 2008. A novel pole figure inversion method: specification of the MTEX algorithm. J. Appl. Cryst. 41, 1024-1037.

Juan, P.A., Berbenni, S., Barnett, M.R., Tomé, C.N., Capolungo, L., 2014. A double inclusion homogenization scheme for polycrystals with hierarchal topologies: application to twinning in $\mathrm{Mg}$ alloys. International Journal of Plasticity 60, 182-196.

Kalidindi, S.R., 2001. Modeling anisotropic strain hardening and deformation textures in low stacking fault energy fcc metals. International Journal of Plasticity 17, 837-860.

Knezevic, M., Zecevic, M., Beyerlein, I.J., Bingert, J.F., McCabe, R.J., 2015. Strain rate and temperature effects on the selection of primary and secondary slip and twinning systems in HCP Zr. Acta Materialia 88, 55-73.

Knezevic, M., Daymond, M.R., Beyerlein, I.J., 2016. Modeling discrete twin lamellae in a microstructural framework. Scripta Materialia 121, 84-88.

Arul Kumar, M., Kanjarla, A.K., Niezgoda, S.R., Lebensohn, R.A., Tomé, C.N., 2015. Numerical study of the stress state of a deformation twin in magnesium. Acta Materialia 84, 349-358.

Lebensohn, R.A., Tomé, C.N., 1993. A self-consistent anisotropic approach for the simulation of plastic deformation and texture development of polycrystals: Application to zirconium alloys. Acta Metallurgica et Materialia 41, 2611-2624.

Lebensohn, R., Uhlenhut, H., Hartig, C., Mecking, H., 1998. Plastic flow of $\gamma$-TiAl-based polysynthetically twinned crystals: micromechanical modeling and experimental validation. Acta mater. 46, 4701-4709.

Lebensohn, R.A., Anand K. Kanjarla, A.K., Philip Eisenlohr, P., 2012. An elasto-viscoplastic formulation based on fast Fourier transforms for the prediction of micromechanical fields in polycrystalline materials. International Journal of Plasticity 32-33, 59-69.

Mareau, C., Daymond, M.R., 2011. Comparison of experimentally determined texture development in Zircaloy-2 with predictions from a rate-dependent polycrystalline model. Materials Science and Engineering A 528, 8676-8686.

Méric, L, Cailletaud, G., 1991. Single crystal modeling for structural calcula- 
tions: Part 2 - Finite element implementation. ASME, Journal of Engineering Materials and Technology 113, 171-182.

Moulinec, H., Suquet, P., 1998. A numerical method for computing the overall response of nonlinear composites with complex microstructure. Computer Methods in Applied Mechanics and Engineering 157, 69-94.

Müller, W.H., 1996. Mathematical versus experimental stress analysis of inhomogeneities in solids. Journal of Physique IV 6, C1.139-C1.148.

Niezgoda, S.R., Kanjarla, A.K., Beyerlein, I.J., Tomé, C.N., 2014. Stochastic modeling of twin nucleation in polycrystals: An application in hexagonal close-packed metals. International Journal of Plasticity 56, 119-138.

Oliver, E.C., Daymond, M.R., Withers, P.J., 2004. Interphase and intergranular stress generation in carbon steels. Acta Materialia 52, 1937-1951.

Proust, G., Tomé, C.N., Kaschner, G.C., 2007. Modeling texture, twinning and hardening evolution during deformation of hexagonal materials. Acta Materialia 55, 2137-2148.

Proust, G., Tomé, C.N., Jain, A., Agnew, S.R., 2009. Modeling the effect of twinning and detwinning during strain-path changes of magnesium alloy AZ31. International Journal of Plasticity 25, 861-880.

Rashid, M.M., Nemat-Nasser, S., 1990. A constitutive algorithm for rate dependent crystal plasticity, Comput. Methods Appl. Mech. Engrg. 94, 201228.

Remy, L., 1981. Metall. Trans. A, 12A, 387.

Robert, C., Mareau, C., 2015. A comparison between different numerical methods for the modeling of polycrystalline materials with an elastic-viscoplastic behavior. Computational Materials Science 103, 134-144.

Simmons, G., Wang, H., 1971. Single Crystal Elastic Constants and Calculated Aggregate Properties: A Handbook, Second Edition, The MIT Press, Cambridge, MA.

Shanthraj, P., Eisenlohr, P., Diehl, M., Roters, F., 2015. Numerically robust spectral methods for crystal plasticity simulations of heterogeneous materials. International Journal of Plasticity 66, 31-45.

Shiekhelsouk, M.N., Favier, V., Inal, K., Cherkaoui, M., 2009, Modelling the behaviour of polycrystalline austenitic steel with twinning-induced plasticity effect. International Journal of Plasticity 25, 105-133.

Staroselsky, A., Anand, L., 2003. A constitutive model for hep materials deforming by slip and twinning: application to magnesium alloy AZ31B. Int. J. Plast. 19, 1843-1864.

Vercammen, S., Blanpain, B., De Cooman, B.C., Wollants, P., 2004. Cold rolling behaviour of an austenitic Fe30Mn3Al3Si TWIP-steel: the importance of deformation twinning. Acta Materialia 52, 2005-2012.

Wu, L., Agnew, S.R., Brown, D.W., Stoica, G.M., Clausen, B., Jain, A., Fielden, D.E., Liaw, P.K., 2008. Internal stress relaxation and load redistribution during the twinning-detwinning-dominated cyclic deformation of a wrought magnesium alloy, ZK60A. Acta Materialia 56, 3699-3707.

Xu, F., Holt, R.A., Daymond, M.R., 2009. Modeling texture evolution during 
uni-axial deformation of Zircaloy-2. Journal of Nuclear Materials 394, 9-19. Zecevic, M., Knezevic, M., Beyerlein, I.J., Tomé, C.N., 2015. An elasto-plastic self-consistent model with hardening based on dislocation density, twinning and de-twinning: Application to strain path changes in HCP metals. Materials Science and Engineering A 638, 262-274.

Zhang, R.Y., Daymond, M.R., Holt, R.A., 2008. A finite element model of deformation twinning in zirconium, Mater. Sci. Eng. A 473, 139-146. 